ARTICLE

Received 20 Aug 2013 | Accepted 7 Nov 2013 | Published 6 Dec 2013

DOI: $10.1038 /$ ncomms3895

\title{
Adaptive strong-field control of chemical dynamics guided by three-dimensional momentum imaging
}

\author{
E. Wells ${ }^{1}$, C.E. Rallis ${ }^{1}$, M. Zohrabi ${ }^{2}$, R. Siemering ${ }^{3}$, Bethany Jochim , P.R. Andrews ${ }^{1}$, U. Ablikim², B. Gaire ${ }^{2, \dagger}$, \\ S De ${ }^{2, \dagger}$, K.D. Carnes ${ }^{2}$, B. Bergues ${ }^{4}$, R. de Vivie-Riedle ${ }^{3}$, M.F. Kling ${ }^{2,4}$ \& I. Ben-Itzhak ${ }^{2}$
}

Shaping ultrafast laser pulses using adaptive feedback can manipulate dynamics in molecular systems, but extracting information from the optimized pulse remains difficult. Experimental time constraints often limit feedback to a single observable, complicating efforts to decipher the underlying mechanisms and parameterize the search process. Here we show, using two strong-field examples, that by rapidly inverting velocity map images of ions to recover the three-dimensional photofragment momentum distribution and incorporating that feedback into the control loop, the specificity of the control objective is markedly increased. First, the complex angular distribution of fragment ions from the $n \omega+C_{2} D_{4} \rightarrow C_{2} D_{3}^{+}+D$ interaction is manipulated. Second, isomerization of acetylene $\left(n \omega+\mathrm{C}_{2} \mathrm{H}_{2} \rightarrow \mathrm{C}_{2} \mathrm{H}_{2}^{2+} \rightarrow \mathrm{CH}_{2}^{+}+\mathrm{C}^{+}\right.$) is controlled via a barrier-suppression mechanism, a result that is validated by model calculations. Collectively, these experiments comprise a significant advance towards the fundamental goal of actively guiding population to a specified quantum state of a molecule.

\footnotetext{
${ }^{1}$ Department of Physics, Augustana College, Sioux Falls, South Dakota 57197, USA. ${ }^{2}$ J.R. Macdonald Laboratory, Department of Physics, Kansas State University, Manhattan, Kansas 66506, USA. ${ }^{3}$ Department für Chemie, Ludwig-Maximilians-Universität München, Butenandt-Strasse 11, D-81377 München, Germany. ${ }^{4}$ Max Planck Institute of Quantum Optics, Hans-Kopfermann-Str. 1, D-85748 Garching, Germany. † Present addresses: Lawrence Berkeley National Laboratory, One Cyclotron Road, Berkeley, CA 94720, USA. (B.G.); Saha Institute of Nuclear Physics, 1/AF Bidhannagar, Kolkata 700064, India (S.D.). Correspondence and requests for materials should be addressed to E.W. (email: eric.wells@augie.edu).
} 
C hemical reaction dynamics can be monitored on timescales of the electronic and nuclear motions ${ }^{1-7}$, thanks to advances in laser technology $^{7-9}$. These advances have led to increasing interest in using ultrafast laser pulses to manipulate chemical processes via direct control of the electronic and nuclear dynamics ${ }^{7,10}$. Active manipulation of the dynamics, however, is a challenging problem: rapid internal vibrational redistribution often thwarts simple approaches; the laser-modified potential energy surfaces (PESs) are complex; and the intense lasermolecule interaction must not destroy the target system before it reaches its desired final state. For these reasons and others, a priori selection of suitable laser-pulse characteristics remains an intractable problem for all but the simplest reactions. Adaptive femtosecond control has proved to be a powerful alternative strategy ${ }^{11-14}$

In adaptive femtosecond control, a learning algorithm uses experimental feedback to change the parameters defining the laser pulse shape and thereby iteratively approaches the desired control objective, effectively solving the Schrödinger equation in real time ${ }^{12}$. This approach circumvents the need for detailed knowledge of the nonlinear response of the molecule to the laser field, but the complexity of the resulting optimal pulses often obscures the mechanism driving the control. Disentangling the pulse complexities to arrive at mechanistic insight remains relatively rare (for example refs 14-16), especially in the strongfield regime and in experiments that lack precise spectroscopic feedback signals. Improving the understanding of the control mechanism provides fundamental information about the complex laser-molecule dynamics, thus revealing behaviour that will build the physical intuition necessary to properly parameterize the large multi-dimensional phase space defining the pulse shape ${ }^{17,18}$.

The learning algorithm often needs several thousand trial pulses to converge on a solution, and therefore often a simple product yield (for example, time-of-flight mass spectrometry) is employed to obtain rapid feedback. Although these signals can be obtained quickly, the control target is often ambiguous. Moreover, most successful examples of reverse engineering the pulse characteristics involve the interplay between careful parameterization of the search space and detailed spectroscopic feedback $^{14,15,19}$, whereas similar attempts using ion yields have been less successful ${ }^{18}$. Various multi-dimensional ion imaging techniques can provide spectroscopic level discernment ${ }^{20,21}$, but they have been too slow for adaptive control and have not been implemented so far.

In this Article, we demonstrate the use of a rapid threedimensional (3D) momentum imaging technique that is incorporated directly into the feedback loop for adaptive control of molecular dynamics. Two primary examples are presented. In the first, image-based feedback allows manipulation of the angular distributions of photofragments arising from strong-field ionization of ethylene, a non-linear molecule with a rich spatial pattern. This example demonstrates the ability of $3 \mathrm{D}$ image-based feedback to target and control objectives that are not typically accessible using standard time-of-flight or spectroscopic techniques. In the second example, we control the isomerization dynamics in the doubly charged ions of acetylene. Beyond the control itself, the 3D image-based feedback has stimulated theoretical calculations that identify the barrier-suppression mechanism responsible for increasing the isomerization yield. Furthermore, this control solution has proved robust, with different searches reaching essentially similar pulse characteristics despite different pulse parameterization schemes. On the basis of this range of initial results, we surmise that the use of multidimensional momentum feedback opens new avenues for exploration using adaptive control, especially in complex systems or when spectroscopic feedback is impractical.

\section{Results}

Experiment. As detailed in the Methods section, the image-based feedback was obtained by employing velocity map imaging $(\mathrm{VMI})^{22}$ in combination with a rapid inversion algorithm for online 3D feedback. Although past work has combined strong field pulse shaping with imaging ${ }^{23-25}$, and a few previous efforts $^{26-29}$ used VMI for adaptive control, they were exclusively based on raw images, in which the 3D momentum distributions were projected onto the two-dimensional (2D) detector plane. The azimuthal ambiguity inherent in such raw, 2D images complicated the extraction of a robust feedback signal ${ }^{26}$. Previous inversion methods to obtain slices through the $3 \mathrm{D}$ momentum distribution were often too slow to be practical for adaptive control or depended critically on selecting initial parameters (such as the correct basis set for transformation) before the inversion. By implementing a rapid onion-peeling algorithm $^{30}$, the inversion time for a $1,050 \times 1,024$ pixel image was reduced to less than one second, thus enabling $3 \mathrm{D}$ momentum feedback for adaptive control.

Ethylene. Our first control example applies 3D image-based feedback to strong-field ionization of ethylene, specifically the complex angular dependence of $\mathrm{C}_{2} \mathrm{D}_{3}^{+}$ions produced by deuterium atom elimination $\left(n \omega+\mathrm{C}_{2} \mathrm{D}_{4} \rightarrow \mathrm{C}_{2} \mathrm{D}_{3}^{+}+\mathrm{D}\right)$. This process produces the inner four-lobed structure, shown in Fig. 1a, whereas the outer ring structure has a momentum that matches a similar structure on the $\mathrm{D}^{+}$VMI data, suggesting that it is associated with the double ionization channel $\left(n \omega+\mathrm{C}_{2} \mathrm{D}_{4} \rightarrow\right.$ $\left.\mathrm{C}_{2} \mathrm{D}_{3}^{+}+\mathrm{D}^{+}\right)$. As the lobes associated with $\mathrm{C}_{2} \mathrm{D}_{3}^{+}+\mathrm{D}$ are neither perpendicular nor parallel to the polarization direction, extracting this weak channel from time-of-flight mass spectrometry would be difficult. Even the use of raw, 2D VMI is problematic, as azimuthal contributions from the dominant outer double-ionization ring would obscure the inner structure. The 3D momentum feedback allows extraction of the angle-resolved yield from the regions shown in Fig. 1a, which subsequently define the control objective.

The control objective, $\alpha \equiv N_{\mathrm{I}} / N_{\mathrm{II}}$, was defined in terms of a ratio of the yields $(N)$ in the two regions (labelled I and II) shown in Fig. 1a. Optimizing $\alpha$, or selecting laser pulse shapes to shift the angular distributions towards the polarization direction, led to a $65 \%$ enhancement of the control objective. This increase was 13 times greater than the level of random fluctuations in the experiment $(\sigma)$. The resulting angular distribution is shown in Fig. 1b. Moving the angular distribution away from the polarization angle (that is, optimizing $\alpha^{-1}$ ) as shown in Fig. 1b, proved harder, with an enhancement of $22 \%(6 \sigma)$.

Acetylene. The second example of the 3D image-based control technique involves strong-field isomerization of acetylene dications. Isomerization is monitored by examining the $\mathrm{CH}_{2}^{+}$and $\mathrm{CH}^{+}$ion images from the laser-induced fragmentation of the molecule. Previous coincidence measurements ${ }^{31,32}$ indicated that $\mathrm{CH}_{2}^{+}$ions are associated with a vinylidene-like configuration of the dication, whereas $\mathrm{CH}^{+}$ions are the result of double ionization leading to a symmetric $\mathrm{CH}^{+}+\mathrm{CH}^{+}$breakup from the acetylene configuration. The migration of the hydrogen in the former process imparts a significant angular momentum to the molecule, producing a nearly isotropic distribution of ions ${ }^{32}$. The symmetric breakup of acetylene-like $[\mathrm{HCCH}]^{2+}$, however, occurs predominately along the laser polarization direction. The $\mathrm{CH}_{2}^{+}$and $\mathrm{CH}^{+}$angular distributions obtained in our experiments are displayed in Fig. 2, showing the probability density as a function of both the fragment kinetic energy release (KER) and $\cos \theta$, where $\theta$ is the angle between the ion momentum 
a

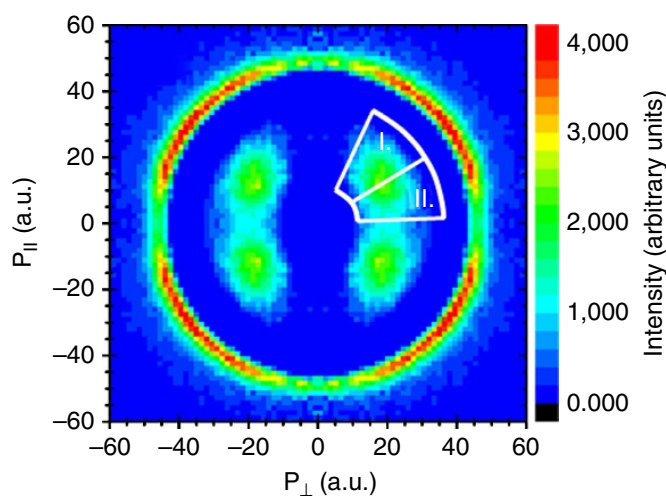

b

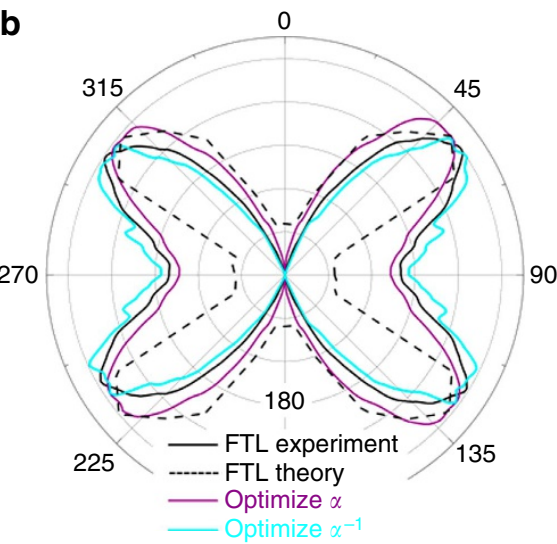

Figure $1 \mid \mathbf{C}_{\mathbf{2}} \mathbf{D}_{\mathbf{3}}^{+}$photofragment image and angular distribution. (a) Measured momentum distribution (in atomic units) of $\mathrm{C}_{2} \mathrm{D}_{3}^{+}$fragments following ionization by near FTL $45 \mathrm{fs}$ duration laser pulses centred at $782 \mathrm{~nm}$ with a peak intensity of $2 \times 10^{15} \mathrm{~W} \mathrm{~cm}^{-2}$. The laser polarization direction is vertical in all panels. The more intense outer structure has a similar shape and momentum to that observed in the $\mathrm{D}^{+} \mathrm{VMI}^{-}$data, and is therefore assumed to be associated with the $\mathrm{C}_{2} \mathrm{D}_{3}^{+}+\mathrm{D}^{+}$channel. The inner structure has no similar momentum matching partner, and is thus associated with $\mathrm{C}_{2} \mathrm{D}_{4}^{+} \rightarrow$ $\mathrm{C}_{2} \mathrm{D}_{3}^{+}+\mathrm{D}$. The white lines define the two regions from which yields were extracted to determine $\alpha$, the control objective. (b) A comparison of the measured angular distributions for strong-field ionization by different optimized pulses leading to $C_{2} D_{4}^{+} \rightarrow C_{2} D_{3}^{+}+D$. Violet represents the distribution obtained by maximizing $\alpha$ and cyan arises from maximizing $\alpha^{-1}$. In these experiments, $\alpha$ increased by $65 \%$ over the value with a FTL pulse. To put this number in context, we note that over the duration of the search process, the fitness value obtained with FTL pulses fluctuated with a standard deviation $(\sigma)$ of $5 \%$ around the average value, and thus the enhancement of $\sigma$ relative to the value obtained with the near-FTL pulse level equates to $13 \sigma . \alpha^{-1}$ was increased by $22 \%$ or $6 \sigma$. In black is the comparison between experimental (solid) and theoretical (dashed) angle-resolved ionization probabilities for the $\mathrm{C}_{2} \mathrm{D}_{4}^{+} \rightarrow \mathrm{C}_{2} \mathrm{D}_{3}^{+}+\mathrm{D}$ process shown in Fig. 1a.
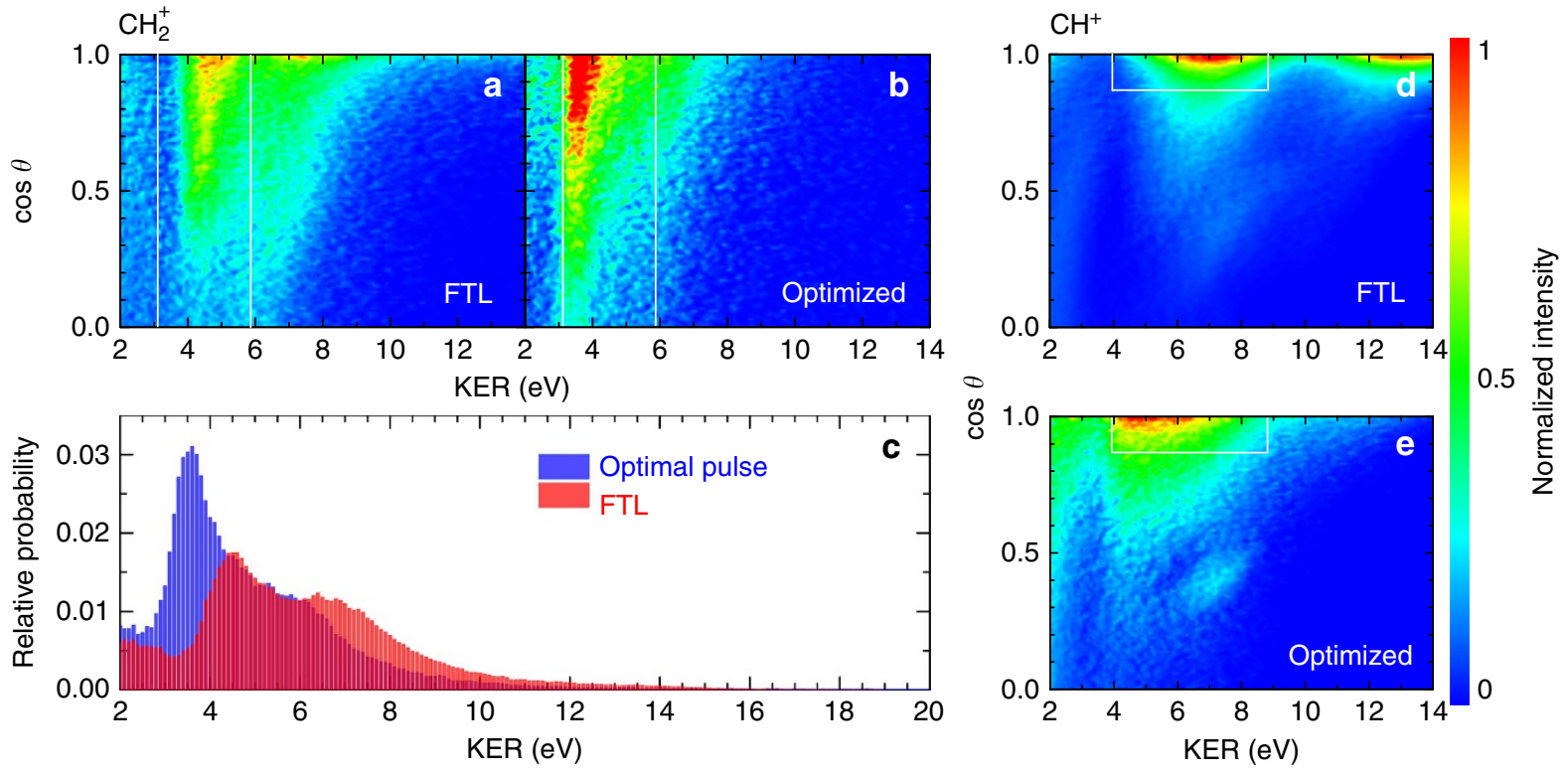

Figure $2 \mid \mathbf{C H}_{\mathbf{2}}^{+}$and $\mathbf{C H}^{+}$photofragment data. Measured density plots of dissociating $\mathrm{CH}_{2}^{+}$(a and $\mathbf{b}$ ) and $\mathrm{CH}^{+}$(d and $\mathbf{e}$ ) ions as a function of $\mathrm{KER}$ and $\cos \theta$ for FTL pulses (a and $\mathbf{d}$ ) and pulses optimized to enhance the $\mathrm{CH}_{2}^{+}$channel ( $\mathbf{b}$ and $\mathbf{e}$ ). The white boxes in the density plots define the regions used to obtain the $\mathrm{CH}_{2}^{+}$and $\mathrm{CH}^{+}$yields used in the control objective. As shown in (c), the probability of dissociation as a function of $\mathrm{KER}_{\text {for }} \mathrm{CH}_{2}^{+}$ ions reveals that laser pulses tailored to enhance the angle-resolved $\mathrm{CH}_{2}^{+} / \mathrm{CH}^{+}$ratio produce $\mathrm{CH}_{2}^{+}$ions with a KER distribution that peaks at more than $1 \mathrm{eV}$ lower than the distribution obtained with FTL pulses. The FTL pulses have a centre wavelength of $778 \mathrm{~nm}$, a FWHM duration of $45 \mathrm{fs}$ and a focused intensity of $\approx 8 \times 10^{14} \mathrm{~W} \mathrm{~cm}^{-2}$. Each density plot is normalized to the peak intensity for that particular exposure and displayed with the same linear colour scale (shown at right) for each image.

and the laser polarization axis. The earlier acetylene work ${ }^{31,32}$ suggested that the regions shown by the white lines in Fig. 2a,b,d,e be used to define the vinylidene/acetylene ratio.

The KER distribution of $\mathrm{CH}_{2}^{+}$ions obtained with a Fouriertransform limited (FTL) pulse is peaked at $4.85 \mathrm{eV}$, consistent with previous coincidence measurements of the $\mathrm{C}^{+}+\mathrm{CH}_{2}^{+}$ dissociative double-ionization channel, which show a sharplypeaked distribution near $5 \mathrm{eV}$ that is nearly independent of $\cos \theta$ (refs 31,32). The $\mathrm{CH}^{+}$fragments peaked at around $7 \mathrm{eV}$ along the polarization direction are consistent with previous 

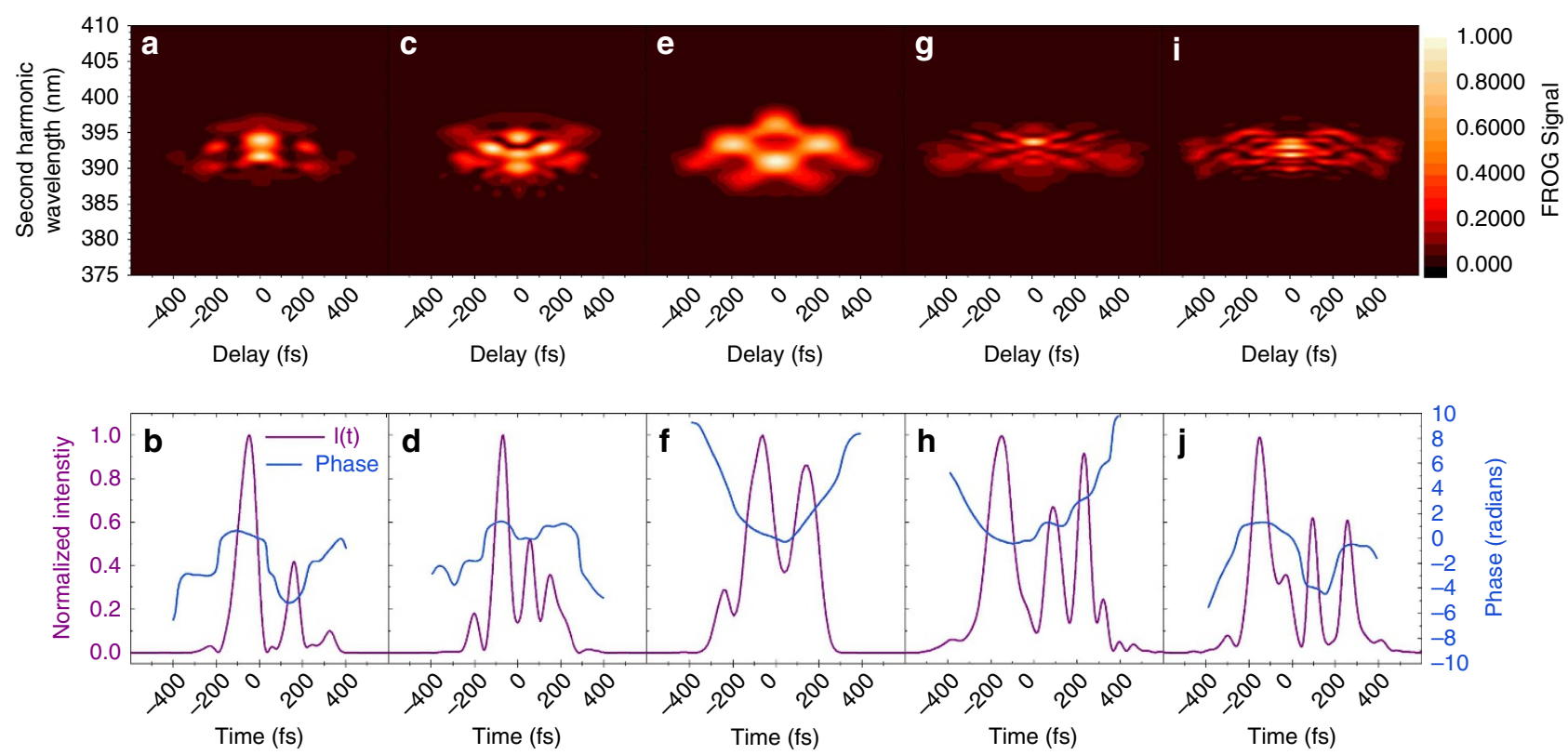

Figure 3 | Laser pulse characterization. SHG FROG ${ }^{30}$ measurements (top) and the associated intensity and temporal phase (bottom) for different experimental trials. (a,b) Optimized pulse using phase-only frequency domain shaping at $68 \mu \mathrm{J}$ per pulse obtaining $55 \%$ enhancement of the $\mathrm{CH}_{2}^{+} / \mathrm{CH}^{+}$ ratio. (c,d) and (e,f) are similar but at $130 \mu \mathrm{J}$ and again at $68 \mu \mathrm{J}$ pulse energy, obtaining $92 \%$ and $44 \%$ enhancement, respectively. (g,h) Results from phase-only time-domain shaping at $60 \mu \mathrm{J}$ per pulse, obtaining $90 \%$ enhancement. The final pulse $(\mathbf{i}, \mathbf{j})$ is manually designed and achieved $40 \%$ enhancement.

measurements of the $\mathrm{C}_{2} \mathrm{H}_{2}^{2+}$ dissociation into $\mathrm{CH}^{+}+\mathrm{CH}^{+}$, whereas the higher KER features are assumed to arise from higher charge states of the parent molecule.

When the learning algorithm was allowed to adjust the pulse shape, the angle-resolved target ratio of $\mathrm{CH}_{2}^{+} / \mathrm{CH}^{+}$could be either almost doubled $(40 \sigma)$ or suppressed to half of the original value $(25 \sigma)$ over a range of laser pulse energies. Interestingly, the KER obtained with the pulse optimized to enhance the isomerization channel showed a downward shift to $3.6 \mathrm{eV}$ and suppression of the high KER feature around $7 \mathrm{eV}$.

As illustrated in Fig. 3, measurement of these optimized pulses with a SHG FROG ${ }^{33}$ showed that the individual pulse characteristics have little similarity aside from a need for multiple peaks spaced by $100-210 \mathrm{fs}$.

\section{Discussion}

The angle-resolved ethylene data, showing increased ionization when the polarization is aligned with the $\mathrm{C}-\mathrm{D}$ bond, gives insight into the ionization process. In a molecular context, the angular distribution of the strong-field ionization events are often understood in terms of the molecular orbitals involved ${ }^{34-39}$. Early theoretical efforts concentrated on ionization from the $\mathrm{HOMO}^{34}$, but later studies showed that when occupied valence orbitals have similar energies, both the HOMO and the HOMO-1 states can contribute to the ionization ${ }^{35-38}$. As the energy gap between orbitals decreases in polyatomic molecules, more orbitals are expected to contribute.

The pattern shown in Fig. 1a can be understood by extending the calculations of angle-resolved ionization rates of diatomic molecules, described in ref. 39 , to polyatomic molecules. The neutral $\mathrm{C}_{2} \mathrm{D}_{4}$ molecules in our effusive jet source are randomly oriented, and thus the interaction between the laser field and the electronic wavefunction depends on their relative angle. When the laser polarization is either parallel or perpendicular to the $\mathrm{C}=\mathrm{C}$ backbone, the HOMO, LUMO and HOMO-1 orbitals are only slightly distorted. When the laser polarization is aligned with the $\mathrm{C}-\mathrm{D}$ bond direction, however, the electron density becomes much more pliable. The result is a strongly distorted Rydberg orbital located near the deuterium atom. In this configuration, the field easily shifts electron density in that direction and the ionization rate correspondingly increases. As shown in Fig. 1b, the measured and calculated angle-resolved ionization probabilities are in reasonable agreement for FTL pulses. From a control perspective, then, modification of the angular distribution of $\mathrm{C}_{2} \mathrm{D}_{3}^{+}$fragments is related to the ability of the pulse to influence the location of this Rydberg orbital. Without image-based feedback, this task would be impossible. Moreover, the $\mathrm{C}_{2} \mathrm{D}_{3}^{+}$ images provide experimental verification of the theoretical predictions suggesting that multiple orbitals, including the distorted Rydberg orbital, have important roles in the ionization of unsaturated hydrocarbons.

In a larger sense, this example is significant because it illustrates the link image-based feedback can provide between tunnelling ionization and molecular structure. The lobe structures in this example should be present at appearance intensities because the tunnelling rates are highest where the electron cloud has the largest extent ${ }^{34}$. Dissociation channels such as $n \omega+\mathrm{C}_{2} \mathrm{D}_{4} \rightarrow \mathrm{C}_{2} \mathrm{D}_{3}^{+}+\mathrm{D}$ that are energetically accessible at the lowest intensity are therefore the best channels for probing and learning how to control molecular structure. Extending this link to the pulse characteristics in this case is still under investigation. Enhancing $\alpha$ was accomplished via a pulse that had some modulation of the spectral intensity but was only slightly longer in duration than the FTL pulse. The pulse that maximized $\alpha^{-1}$ has complicated phase characteristics and a more complex structure.

In the acetylene experiment, however, the image-based feedback stimulated model calculations that yielded an understanding of the mechanism driven by the optimized laser pulse. To shed light on the molecular dynamics and time scales involved, we performed semiclassical on-the-fly calculations in full dimension 
for the doubly charged acetylene ion. The simulation started just after the ionization event and included the $X^{3} \Sigma_{\text {g }}^{-}$ground state and the first singlet state ${ }^{1} \Delta_{\mathrm{g}}$ of $\mathrm{C}_{2} \mathrm{H}_{2}^{2+}$. Interactions with both FTL and optimized laser fields were considered. The details for the full dimension trajectory calculations are given in the Methods section. To visualize the dynamics, the full dimension trajectories have been projected onto a 2D PES spanned by the most significant reaction coordinates. This reaction PES includes all critical points, such as minima and barriers along the pathway, and shows both fragmentation channels used as the defined control objective $\left(\mathrm{CH}_{2}^{+} / \mathrm{CH}^{+}\right)$. The first coordinate corresponds to the $\mathrm{H}$ migration, which can be followed in the $\mathrm{CCH}$ angle. The second coordinate is the $\mathrm{CC}$ distance leading to either the $\mathrm{CH}_{2}^{+}$ $+\mathrm{C}^{+}$or $\mathrm{CH}^{+}+\mathrm{CH}^{+}$fragmentations, depending on the $\mathrm{CCH}$ angle. The $a b$ initio PES calculation, as well as the on-the-fly trajectories, used high level electron correlation theory and is shown in Fig. 4.

To reach $\mathrm{CH}_{2}^{+}+\mathrm{C}^{+}$fragmentation (e), a molecule starting from the linear configuration (a) has to pass the transition state (b). It forms a vinylidene-like local minimum geometry at (c) before dissociating over a second barrier (d) to the fragments (e). The second pathway leading to the $\mathrm{CH}^{+}$-fragments includes mostly the CC-bond elongation and is reached after crossing the barrier (f). Two example trajectories for isomerization, projected onto the 2D surface, are shown in Fig. 4 for calculations with (violet line) and without (white line) the optimized laser field. The white trajectory oscillates for some time around the acetylene-like structure (a) before overcoming the barrier to the vinylidene-like geometry. These types of trajectories do not have enough energy to cross the dissociation barrier and either remain in this configuration or return to (a). The violet trajectory moves very swiftly from geometry (a) over the suppressed barrier (b) to (c) and finally dissociates to (d) without staying long at any given structure.

Most of the more than 1,000 trajectories without the optimized laser interaction stayed at structure (a) or moved between (a) and (c). Between 100 and $210 \mathrm{fs}$, trajectories with geometry (c) had the highest probability. This time period matches the time separation of the sub-pulses in the optimized field, and it appears that the second sub-pulse is timed to interact when most molecules are in configuration (c). The laser interaction shifts the PES, suppressing the dissociation barrier towards the $\mathrm{CH}_{2}^{+}$fragments, allowing molecules with lower kinetic energy to cross. The theoretical KER spectrum, shown in Fig. 5, supports this explanation and shows the same shift to a lower KER distribution as observed experimentally. Furthermore, the $\mathrm{CH}^{+}$fragments also show a shift to lower KER (see Fig. 2d,e), which can be interpreted as a lowering of the barrier between points (a) and (g) in the PES. This barrier, however, is higher than the barrier between (c) and (d), and thus dissociation from the vinylidene configuration is expected to be favored, consistent with the experimental observations.

To further examine this isomerization mechanism, we employed phase-only shaping with a time-domain pulse parameterization $^{18}$, rather than the standard frequency-domain parameterization used in the previously described experiments. In this scheme, our optimal pulse is built out of a series of Gaussian shaped pulses and the parameters controlled by the learning algorithm are the duration, amplitude and separation between the individual pulses. Using this parameterization and a

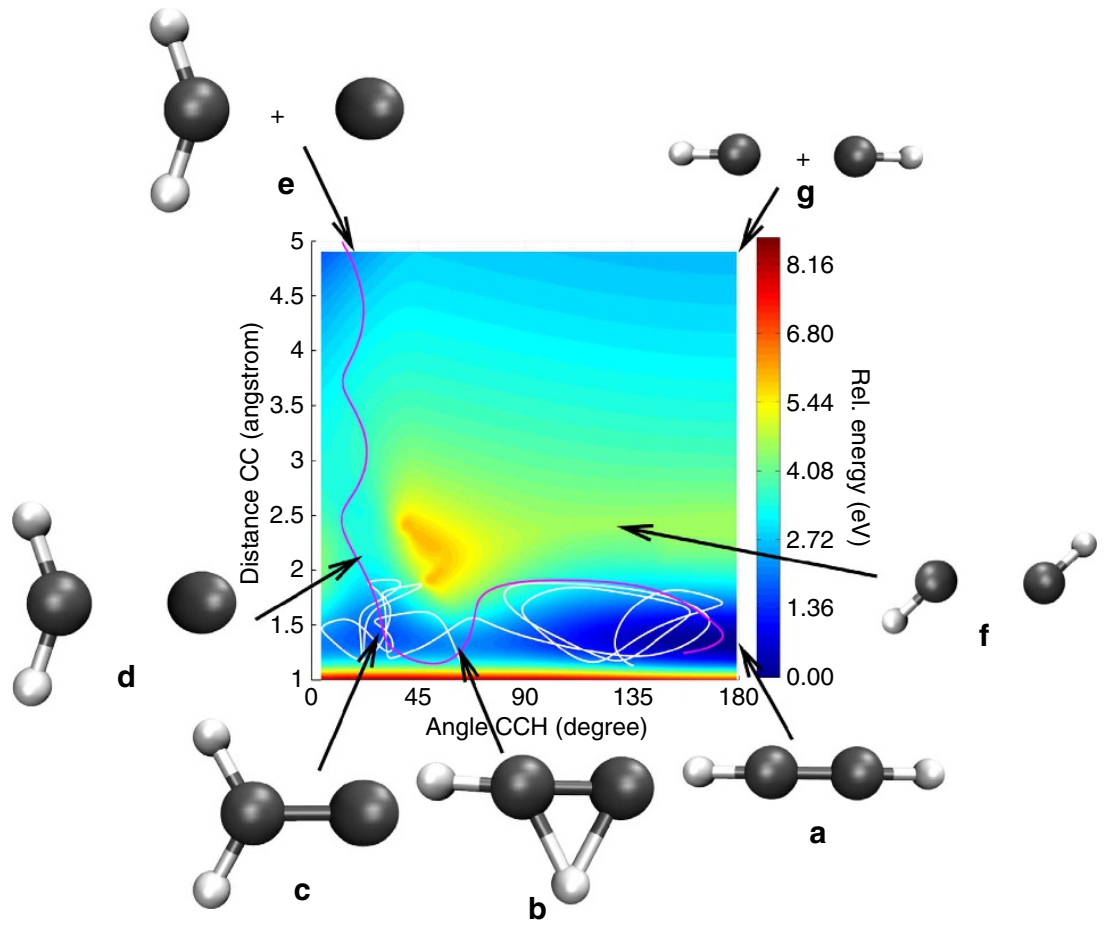

Figure 4 / $\mathbf{C}_{\mathbf{2}} \mathbf{H}_{\mathbf{2}}^{\mathbf{2 +}}$ PES and sample trajectories. The 2D PES of the acetylene dication for the two coordinates $\mathrm{CCH}$ angle and $\mathrm{CC}$ distance. Important geometries (labelled $\mathbf{a}-\mathbf{g}$ ) are marked on the surface with black arrows linked to corresponding ball-and-stick structures. Point (a) on the surface represents the initial linear acetylene state, (b) represents the intermediate transition state and (c) represents the vinylidene configuration of the $\mathrm{C}_{2} \mathrm{H}_{2}^{2+}$ dication. Dissociation from the vinylidene configuration is shown at points (d) and (e), whereas direct dissociation from the acetylene configuration proceeds through points (f) and $(\mathbf{g})$. Two example trajectories are projected onto these coordinates. The white trajectory is calculated without additional laser interaction following the double ionization step and moves only between the structures (a) over (b) to (c). The violet trajectory includes the effect of the optimized laser pulse and progresses from configuration (a) over (b) to (c) and dissociates to (d) and finally (e) because the laser interaction suppresses the barrier in the region near configuration (d). 


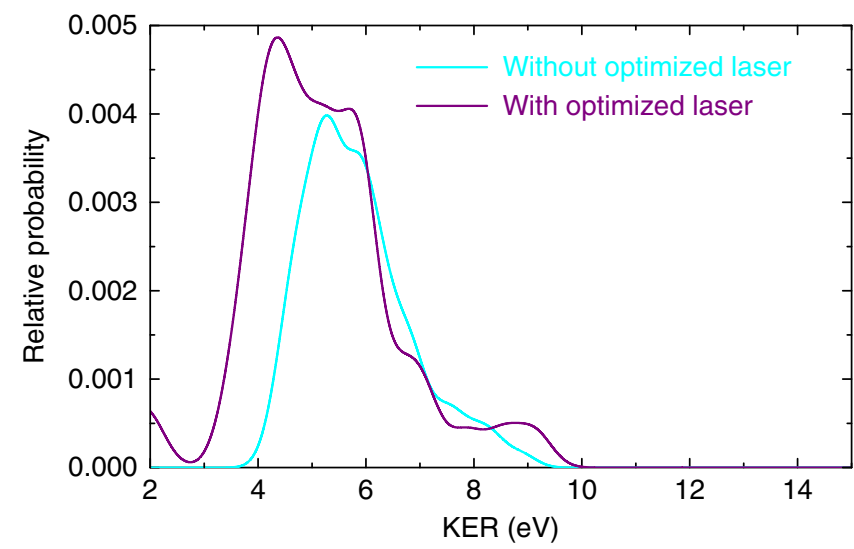

Figure $\mathbf{5}$ | Calculated KER spectrum for $\mathbf{C H}_{\mathbf{2}}^{+}$fragments. In cyan is the probability as a function of KER derived from trajectories evolving without the optimized laser pulse, that is, the calculation assumes that a near-FTL pulse accomplishes the double ionization and the subsequent wavepacket propagation is field-free. In violet is the KER spectrum associated with trajectories evolving in the presence of the optimized laser pulse that modifies the PES. In both the experiment (see Fig. 2c) and calculations, the optimized pulse opens an additional contribution at lower KER.

set of three Gaussian pulses (see Fig. 3g,h), we were again able to double the $\mathrm{CH}_{2}^{+} / \mathrm{CH}^{+}$ratio. The reduced parameter set also converges to a solution much faster (five generations) than the original frequency domain method $(\approx 20$ generations $)$. Importantly, the multiple-pulse solution appears general.

The Dazzler pulse shaper ${ }^{40}$ can also be used to shape the pulse manually by adjusting laser parameters such as the chirp or pulse duration. Investigations along these lines produced some minimal variation in the $\mathrm{CH}_{2}^{+}$to $\mathrm{CH}^{+}$production ratio, but no simple pulse parameter could be manipulated to produce results comparable to those obtained using adaptive control. On the basis of the pulse-train features we observed in pulses optimized to enhance the isomerization ratio in acetylene, we manually designed a pulse with these important characteristics. Although it exceeded the transform-limited result, our pulse did not perform as well as the algorithm-based optimal pulse. The manuallyconstructed pulse train, shown in Fig. 3i,j), produced a $\mathrm{CH}_{2}^{+}$/ $\mathrm{CH}^{+}$ratio $40 \%$ higher than the FTL value at $60 \mu \mathrm{J} /$ pulse, whereas various optimized pulses reached $90 \%$ or a greater enhancement of the ratio. Still, this result demonstrates that the 3D VMI-based feedback contains enough clues about the control dynamics to interpret the essential features of the pulse, even without using complicated numerical methods such as principal control analysis ${ }^{18,41,42}$. This allowed the selection of a reduced parameter set that provided as much control as the larger original basis. Gaining enough mechanistic insight to understand how to choose a useful parameter space is a key step for improving control fidelity.

To verify the need for 3D momentum imaging feedback for achieving this result, we have performed the acetylene experiment using regions of the images designed to mimic a mass spectrometer with a small solid angle. In these experiments, an enhancement of the $\mathrm{CH}_{2}^{+} / \mathrm{CH}^{+}$ratio was observed, but the result was an angular discrimination artifact, similar to what has been observed for the strong-field dissociative ionization of $\mathrm{CO}^{26}$. The use of the full momentum imaging as a feedback for the coherent control algorithm was thus crucial in finding the barriersuppression mechanism.

Some of the most exciting examples of adaptive control (for example refs $14,15,43,44)$ depend on very specific optical feedback. Collectively, the examples described above show that 3D momentum imaging feedback brings similar capabilities to ion-based experiments. The twin benefits of this approach are illustrated in our experiments: first, 3D image-based control allows the selection of well-defined control targets; second, the abundant information contained in the feedback signal can give insight into the control mechanism. In the acetylene example, the adaptive search persistently arrived at the multi-pulse barriersuppression isomerization mechanism despite different initial parameterizations of the search space. This insensitivity to the initial parameterization indicates a high degree of control fidelity. The ethylene examples illustrate how angle-resolved feedback is crucial for the control of polyatomic systems. The general applicability of full momentum-imaging-based feedback for the adaptive control of chemical reaction dynamics promises to uncover novel control mechanisms for even more complex chemical reactions. Furthermore, strong-field processes in atoms, clusters and nanoparticles may be selectively controlled, and the resulting images may provide richly detailed information about the control mechanisms.

\section{Methods}

General experimental details. The core of the experiment was a closed-loop system that produces shaped, intense ultrafast laser pulses, which ionize and fragment the target molecules, producing a feedback that drives the optimization of the pulse shapes. The intensity of these pulses was sufficient to ionize either acetylene or ethylene multiple times and produce transient modifications of their PESs. The resulting photofragments were imaged with a VMI apparatus. This ion image was processed online (as described later) and a particular 'fitness' value was extracted, which was used by the learning algorithm to produce a new set of pulse shapes for testing. The Dazzler pulse shaper may be operated in modes that shape either the spectral phase or amplitude independently or together. In these experiments, we only shaped the phase, and thus the pulse energies remained approximately constant over the duration of an experiment. The system was linked by a computer control that connected the AOPDF pulse shaper, the learning algorithm, the digital delay generator that triggered the high-voltage switch for the microchannel plates (MCPs) and the camera readout electronics. This is illustrated schematically in Fig. 6.

Rapid inversion. In this control scheme, accurate feedback from the velocity map image, in the form of an ion yield from a particular portion of the image, often depends critically on the conversion of the $2 \mathrm{D}$ raw image to a slice through the centre of the full 3D momentum distribution. An example is displayed in Fig. 7, which shows the raw and inverted images of $\mathrm{CD}_{3}^{+}$ions produced in a laserethylene interaction. Although the outer structure is visible in the raw image on the left, the inner structure is nearly completely obscured by an unwanted azimuthal contribution that can easily lead to sub-optimal control results ${ }^{26}$.

Mathematically, given the cylindrical symmetry conveniently provided by the laser polarization axis, accomplishing the image inversion requires performing an inverse Abel transformation ${ }^{22,45-47}$. A number of numerical methods for solving this problem exist, but not all of them are useful for this rapid online inversion owing to the additional constraints of the adaptive control loop. First, as a typical experiment contains a few thousand trial pulses, the inversion cannot take much longer than the image acquisition time. Ideally, the inversion time will be much shorter. Second, as the image features are not known ahead of time, methods that require manual selection of initial parameters (such as an appropriate basis set) are difficult to implement. Finally, the inversion process should avoid introducing noise that compromises the feedback.

Inversion selection and speed tests. With these constraints in mind, it is worth reviewing a few options. Fourier-Hankel-based methods ${ }^{48,49}$ can be quite rapid but can often result in increased noise, especially near the centre of the image where the Bessel function oscillates rapidly ${ }^{22}$. Several methods, such as BASEX ${ }^{50}$ and pBASEX $^{51}$, take advantage of the fact that some functions have known inverse Abel transformations. These methods have several advantages, including a relocation of the centerline noise common to the Abel methods to the centre point of the image, relatively rapid computation and smooth final images. The main drawback for our application is that they require the selection of a basis set before the experiment. An alternative approach, which converges to the measured $2 \mathrm{D}$ projection by making iterative guesses about the $3 \mathrm{D}$ distribution, was developed by Vrakking ${ }^{52}$ but is significantly slower. In recent years, several hybrid methods have appeared, including a polar onion peeling (POP) ${ }^{53}$ approach that uses a basis set technique similar to BASEX, and an approach that combines BASEX-like features with the iterative approach ${ }^{54}$. These also had undesirable features for our online processing application, primarily the need to set initial values of some kind. In addition, the 


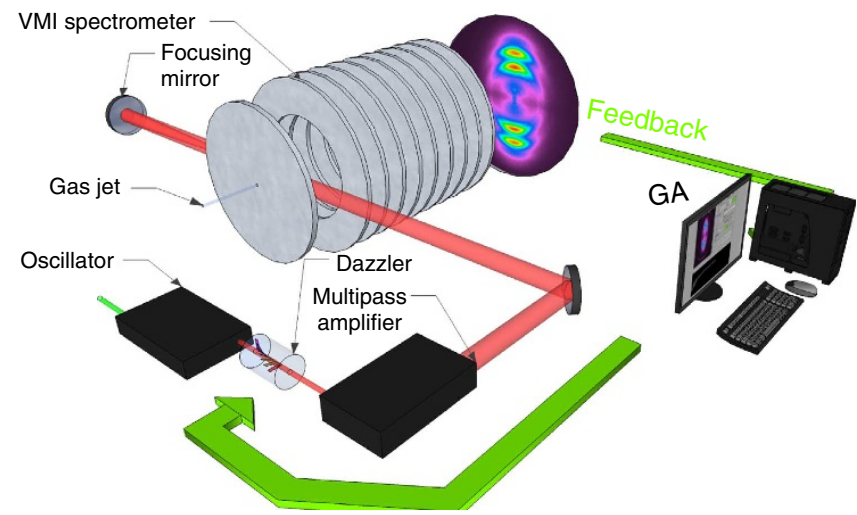

Figure 6 | Schematic diagram of the apparatus and the control loop. Laser pulses centered at approximately $780 \mathrm{~nm}$ were produced with a Ti:sapphire oscillator, shaped with an acousto-optical programmable dispersive filter ${ }^{37}$ and then amplified to energies typically between 0.01 and $0.5 \mathrm{~mJ}$ per pulse by a multipass amplifier running at $2 \mathrm{kHz}$. The near-FTL pulses had durations of approximately $40-45 \mathrm{fs}$ and, after they were focused by a $(f=75 \mathrm{~mm})$ spherical mirror, achieved peak intensities of approximately $2 \times 10^{16} \mathrm{~W} \mathrm{~cm}^{-2}$. The pulses intersected an effusive jet of target gas, similar to the one described in ref. 42. The spectrometer electric fields were configured so that trajectories corresponding to a particular initial velocity arrived at the same position on the detector plane, even if the ions had been created at different locations within the laser focus ${ }^{43}$. The ions struck the detector MCP stack, producing an electron shower on a phosphor screen that was imaged by a CCD camera. The MCP assembly was fully powered only at times corresponding to the arrival of a single preselected ion species using a triggered high-voltage switch. The image was processed and the feedback delivered to the learning algorithm, which created the next set of pulse shapes to test.

POP algorithm produced negative values in our tests, which significantly complicates the determination of a yield-based fitness value.

For these reasons, we selected the well-known 'onion-peeling' or 'backprojection' method ${ }^{22,30}$. Although this technique does result in some centerline noise, it is typically less significant than with the Fourier-Hankel methods, and the noise can be reduced to minimal levels. Importantly, there are no initial parameters to be specified, other than the centre and size of the image. Our implementation was derived from the algorithm described by Bordas and co-workers ${ }^{30}$, and the interested reader is directed to their excellent development of the mathematical formalism. Careful optimization of our implementation of this method reduced the computation time for our $661 \times 661$ pixel test image to $50 \mathrm{~ms}$, shorter than any other method we tested. The overall image processing time is typically less than the duration of the image acquisition, and therefore the overall time of the experiment is not affected.

Pulse parameterization. In most of these experiments the spectral phase was controlled via sixteen 'genes' spread over the pulse bandwidth. The spectral phases between adjacent gene values are calculated by linear interpolation. The timedomain parameterization method allows the shaped pulse to be built out of a series of $n$ Gaussian-shaped pulses ${ }^{18}$. In an example where the full pulse is composed of three component pulses, eight genes are needed: three amplitudes $\left(a_{1}, a_{2}\right.$ and $\left.a_{3}\right)$, three pulse durations $\left(w_{1}, w_{2}\right.$ and $\left.w_{3}\right)$ and two time delays $\left(t_{12}\right.$ and $\left.t_{13}\right)$. To obtain the phase mask needed to produce the desired laser intensity as a function of time while only controlling the spectral phase delay, we used the algorithm developed by Hacker and co-workers ${ }^{55}$. This algorithm rapidly estimates the needed phase mask using a process that iteratively employs a FFT-based approach.

FTL fitness determination. The degree of control is reported with respect to the value of the fitness function obtained with a FTL pulse. Experimentally, between each generation an FTL pulse is tested under the same conditions as the trial pulse. These results are averaged over the entire experiment to produce the baseline value that serves as a comparison for the shaped pulses. The fluctuations in the FTL fitness value obtained during each experiment define $\sigma$ for that experiment.

Selection of regions of interest. Generally, images of various fragmentation channels are obtained with FTL pulses and these results are used to make an initial selection of a feature for optimization. In the acetylene example, previous

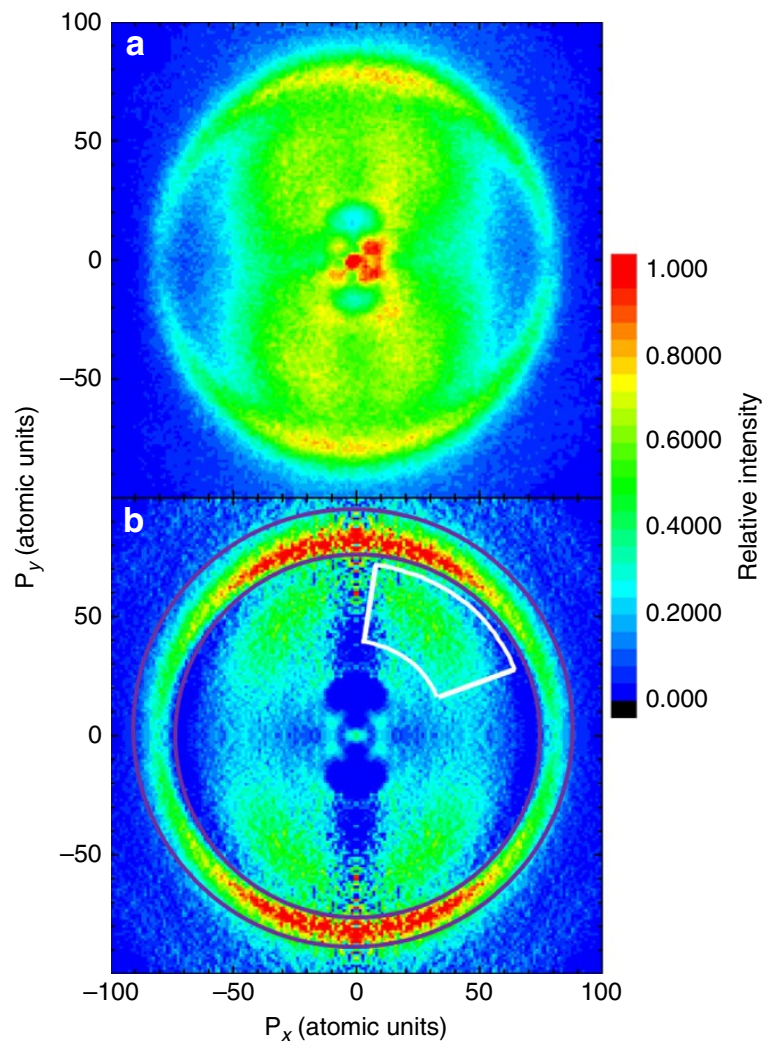

Figure 7 | Comparison of raw 2D and processed 3D images. The raw (a) and rapidly inverted (b) images for $\mathrm{CD}_{3}^{+}$ions. The inversion was accomplished with the 'onion-peeling' or 'back-projection' method described in this section. The purple and white boxed regions in (b) illustrate how different processes could be isolated from the $\mathrm{CD}_{3}^{+}$images and the corresponding yields used to define a control objective. On a $661 \times 661$ pixel test image, our algorithm could produce an inverted image in $50 \pm 5 \mathrm{~ms}$, compared with $1.5 \mathrm{~s}$ for pBASEX $51,4.86 \mathrm{~s}$ for POP 53 and $174 \mathrm{~s}$ for the iterative procedure ${ }^{52}$. For a full-sized $1040 \times 1054$ pixel image, the inversion time was still only around $600 \mathrm{~ms}$, well below the image acquisition time. Additional efficiency was gained by separating the image acquisition and analysis steps. Thus, while one image was being acquired the previous image was being inverted. As the exposure time is almost always longer than the image processing time, the overall time for the experiment was not affected by the addition of the inversion step into the control loop.

results ${ }^{31,32}$ were also used to inform our selection. Features will often shift in energy or angular distribution as different laser pulse shapes are applied, so selecting too restrictive of a region for use in calculating the fitness function can lead to difficulties with the search. In fact, too restrictive of a gate on the $\mathrm{CH}_{2}^{+}$ image shown in Fig. 2a,b did lead to spurious results by excluding lower KER ions from the fitness calculation. In this particular case, however, extending the gate lower in KER than shown in Fig. 2a,b did not affect the results.

Ethylene calculations. As described more fully in ref. 39, the angular-dependent ionization probabilities for ethylene are calculated based on the electronic structure theory. To describe the interaction between the laser pulse and the molecule, the polarization is fixed and the molecule is rotated around that plane as well as out of the plane, that is, around the $\mathrm{C}=\mathrm{C}$ bond direction. For each position, the electronic wave function is calculated in the presence of the electric field and the tunnelling probability is deduced for the various orbitals. Ionization from more than a single molecular orbital is treated using a linear combination of the selected orbitals.

Acetylene calculations. The $2 \mathrm{D}$ PES of $\mathrm{C}_{2} \mathrm{H}_{2}^{2+}\left(\mathrm{X}^{3} \Sigma_{\mathrm{g}}^{-}\right)$was calculated on the CASSCF $^{8,10}$ level with a $6-311++\mathrm{G}^{* *}$ basis set using the programme package MOLPRO $^{56}$. The scan was carried out under relaxation of the remaining coordinates. The acetylene trajectories were calculated on the same level of theory 
and utilized an adapted version of the NewtonX program package ${ }^{57}$ for the on-thefly interface to MOLPRO. Trajectories were calculated for the $\mathrm{X}^{3} \Sigma_{\mathrm{g}}^{-}$and $1^{1} \Delta_{\mathrm{g}}$ states. They show similar topography and can be reached in the ionization process. To include the effect of the remaining laser field after ionization, we first checked for possible interaction pathways via electronic excited states that could be used for laser-based control. No electronic excited states can be reached with the laser frequency (or are symmetry forbidden) for both the singlet and the triplet states of the dication. The possibility of two photon transitions was also considered following the approach of ref. 58, but could be excluded. For the FTL laser pulse we assumed a Wigner distribution in the Franck-Condon region of the molecule with excess energy from the laser distributed in the vibrational modes ${ }^{59,60}$. Here, we assumed that the first half of the laser pulse is needed for ionization. For the optimal laser pulse, we took the experimental field and assumed that ionization occurred around the maximum of the first sub-pulse. After ionization the trajectories started again from individual points of a Wigner distribution in the Franck-Condon region. The remaining laser pulse was then added to the oneelectron Hamiltonian at the distinct time steps and was included in the on-the-fly dynamics.

\section{References}

1. Zewail, A. H. Femtochemistry: Atomic-Scale Dynamics of the Chemical Bond Using Ultrafast Lasers (Nobel Lecture). Angew. Chem. Int. Ed. 39, 2586-2631 (2000).

2. Baker, S. et al. Probing Proton Dynamics in Molecules on an Attosecond Time Scale. Science 312, 424-427 (2006).

3. Lee, H., Cheng, Y.-C. \& Fleming, G. R. Coherence Dynamics in Photosynthesis: Protein Protection of Excitonic Coherence. Science 316, 1462-1465 (2007).

4. Carbone, F., Kwon, O.-H. \& Zewail, A. H. Dynamics of Chemical Bonding Mapped by Energy-Resolved 4D Electron Microscopy. Science 325, 181-184 (2009).

5. Breusing, M., Ropers, C. \& Elsaesser, T. Ultrafast Carrier Dynamics in Graphite. Phys. Rev. Lett. 102, 086809 (2009).

6. Wörner, H. J., Bertrand, J. B., Kartashov, D. V., Corkum, P. B. \& Villeneuve, D. M. Following a chemical reaction using high-harmonic interferometry. Nature 466, 604-607 (2010).

7. Krausz, F. \& Ivanov, M. Y. Attosecond Physics. Rev. Mod. Phys. 81, 163-234 (2009).

8. Goulielmakis, E. et al. Real-time observation of valence electron motion. Nature 466, 739-743 (2010).

9. Monmayrant, A., Weber, S. \& Chatal, B. A newcomer's guide to ultrashort pulse shaping and characterization. J. Phys. B At. Mol. Opt. Phys. 43, 103001 (2010).

10. Brixner, T. \& Gerber, G. Quantum control of gas-phase and liquid phase femtochemistry. ChemPhysChem. 4, 418-438 (2003).

11. Brif, C., Chakrabarti, R. \& Rabitz, H. Control of quantum phenomena: past, present and future. New J. Phys. 12, 075008 (2010).

12. Judson, R. S. \& Rabitz, H. Teaching lasers to control molecules. Phys. Rev. Lett. 68, 1500-1503 (1992).

13. Assion, A. et al. Control of chemical reactions by feedback-optimized phaseshaped femtosecond laser pulses. Science 282, 919-922 (1998).

14. Herek, J. L., Wohlleben, W., Cogdell, R. J., Zeidler, D. \& Motzkus, M. Quantum control of energy flow in light harvesting. Nature 417, 533-535 (2002).

15. Daniel, C. et al. Deciphering the reaction dynamics underlying optimal control laser fields. Science 299, 536-539 (2003).

16. Trallero, C., Pearson, B. J., Weinacht, T., Gillard, K. \& Matsika, S. Interpreting ultrafast molecular fragmentation dynamics with $a b$ initio electronic structure calculations. J. Chem. Phys. 128, 124107 (2008).

17. Roslund, J. \& Rabitz, H. Experimental quantum control landscapes: Inherent monotonicity and artificial structure. Phys. Rev. A 80, 013408 (2009).

18. Wells, E. et al. Closed-loop control of intense-laser fragmentation of $\mathrm{S}_{8}$. Phys. Rev. A 72, 063406 (2005).

19. Hornung, T., Meier, R. \& Motzkus, M. Optimal control of molecular states in a learning loop with a parameterization in frequency and time domain. Chem. Phys. Lett. 326, 445-453 (2000).

20. Ullrich, J., Moshammer, R., Dorn, A., Dörner, R., Schmidt, L.Ph.H. \& SchmidtBöcking, H. Recoil-ion and electron momentum spectroscopy: reactionmicroscopes. Rep. Prog. Phys 66, 1463-1545 (2003).

21. Vredenborg, A., Roeterdink, W. G. \& Jannssen, M. H. M. A photoelectronphotoion coincidence imaging apparatus for femtosecond time-resolved molecular dynamics with electron time-of-flight resolution of $\sigma=18$ ps and energy resolution $\Delta E / E=3.5 \%$. Rev. Sci. Instrum. 79, 063108 (2008).

22. Whitaker, B. J. Imaging in Molecular Dynamics: Technology and Applications (Cambridge University Press, 2003).

23. Geißler, D., Rozgonyi, T., González-Vázquez, González, L., Marquetand, P. \& Weinacht, T. C. Pulse-shape-dependent strong-field ionization viewed with velocity-map imaging. Phys. Rev. A 84, 053422 (2011).
24. Irimia, D. \& Janssen, M. H. M. Toward elucidating the mechanism of femtosecond pulse shaping control in photodynamics of molecules by velocity map photoelectron and ion imaging. J. Chem. Phys. 132, 234302 (2010).

25. Natan, A. et al. Quantum control of photodissociation by manipulation of bond softening. Phys. Rev. A 86, 043418 (2012).

26. Jochim, B. et al. Velocity map imaging as a tool for gaining mechanistic insight from closed-loop control studies of molecular fragmentation. Phys. Rev. A 83, 043417 (2011).

27. Chen, G.-Y., Wang, Z. W. \& Hill, III W. T. Adaptive control of the $\mathrm{CO}_{2}$ bending vibration: Deciphering field-system dynamics. Phys. Rev. A 79, 011401(R) (2009).

28. Krug, M. et al. Coherent strong-field control of multiple states by a single chirped femtosecond laser pulse. New J. Phys. 11, 105051 (2009).

29. Ghafur, O., Rouzée, A., Gijsbertsen, A., Siu, W. K., Stolte, S. \& Vrakking, M. J. J. Impulsive orientation and alignment of quantum-state-selected $\mathrm{NO}$ molecules. Nat. Phys. 5, 289-293 (2009).

30. Bordas, C., Faulig, F., Helm, H. \& Huestis, D. L. Photoelectron imaging spectrometry: Principle and inversion method. Rev. Sci. Instrum. 67, 2257-2268 (1996).

31. Alnaser, A. S. et al. Momentum-imaging investigations of the dissociation of $\mathrm{D}_{2}^{+}$and the isomerization of acetylene to vinylidene by intense short laser pulses. J. Phys. B At. Mol. Opt. Phys. 39, S485-S492 (2006).

32. Hishikawa, A., Matsuda, A., Fushitani, M. \& Takahashi, E. J. Visualizing Recurrently Migrating Hydrogen in Acetylene Dication by Intense Ultrashort Laser Pulses. Phys. Rev. Lett. 99, 258302 (2007).

33. Trebino, R. Frequency-Resolved Optical Gating: The Measurement of Ultrashort Laser Pulses (Kluwer Academic, 2002).

34. Tong, X.-M., Zhao, Z. \& Lin, C. D. Theory of molecular tunneling ionization. Phys. Rev. A 66, 033402 (2002).

35. Alnaser, A. S. et al. Effects of molecular structure on ion disintegration patterns in ionization of $\mathrm{O}_{2}$ and $\mathrm{N}_{2}$ by short laser pulses. Phys. Rev. Lett. 93, 113003 (2004).

36. McFarland, B. K. et al. High Harmonic Generation from Multiple Orbitals in $\mathrm{N}_{2}$. Science 322, 1232-1235 (2008).

37. Znakovskaya, I. et al. Attosecond control of electron dynamics in carbon monoxide. Phys. Rev. Lett. 103, 103002 (2009).

38. Smirnova, O. et al. High harmonic interferometry of multi-electron dynamics in molecules. Nature 460, 972-977 (2009).

39. Von den Hoff, P. et al. Effects of multi orbital contributions in the angulardependent ionization of molecules in intense few-cycle laser pulses. Appl. Phys. B 98, 659-666 (2010).

40. Verluise, F., Laude, V., Cheng, Z., Spielmann, C. H. \& Tournois, P. Amplitude and phase control of ultrashort pulses by use of an acousto-optic programmable dispersive filter: pulse compression and shaping. Opt. Lett. 25, 575-577 (2000).

41. White, J. L., Pearson, B. J. \& Bucksbaum, P. H. Extracting quantum dynamics from genetic learning algorithms through principal control analysis. J. Phys. B At. Mol. Opt. Phys. 37, L399-L405 (2004).

42. Jolliffe, I. T. Principal Component Analysis, 2nd edn. (Springer, 2002).

43. Kuroda, D. G., Singh, C. P., Peng, Z. \& Kleiman, V. D. Mapping excited-state dynamics by coherent control of a dendrimer's photoemission efficiency. Science 326, 263-267 (2009).

44. Roth, M. et al. Quantum Control of Tightly Competitive Product Channels. Phys. Rev. Lett. 102, 253001 (2009).

45. Abel, N. H. Oeuvres Completes. (eds Sylow, E. \& Lie, S.) (Johnson Reprint Corp., 1988).

46. Dasch, C. R. One-dimensional tomography: a comparison of Abel, onionpeeling, and filtered backprojection methods. App. Opt. 31, 1146-1152 (1992).

47. Bracewell, R. N. The Fourier Transform and its Applications (McGraw-Hill, 1978).

48. Smith, L. M. \& Keefer, D. R. Abel inversion using transform techniques. J. Quant. Spectrosc. Radiat. Transfer 39, 367-373 (1988).

49. Castleman, W. Digital Image Processing (Prentice-Hall, 1979).

50. Dribinski, V., Ossadtchi, A., Mandelshtam, V. A. \& Reisler, H. Reconstruction of Abel-transformable images: The Gaussian basis-set expansion Abel transform method. Rev. Sci. Instrum 73, 2634-2642 (2002).

51. Garcia, G. A., Nahon, L. \& Powis, I. Two-dimensional charged particle image inversion using a polar basis function expansion. Rev. Sci. Instrum 75, 4989-4996 (2004).

52. Vrakking, M. J. J. An iterative procedure for the inversion of two-dimensional ion/photoelectron imaging experiments. Rev. Sci. Instrum. 72, 4084-4089 (2001).

53. Roberts, G., Nixon, J., Lecointre, J., Wrede, E. \& Verlet, J. Toward real-time charged-particle image reconstruction using polar onion-peeling. Rev. Sci. Instrum. 80, 053104 (2009).

54. Renth, F., Riedel, J. \& Temps, F. Inversion of velocity map ion images using iterative regularization and cross validation. Rev. Sci. Instrum. 77, 033103 (2006). 
55. Hacker, M., Stobrawa, G. \& Feurer, T. Iterative Fourier transform algorithm for phase-only pulse shaping. Opt. Express 9, 191-199 (2001).

56. Werner, H.-J. et al. MOLPRO, version 2006.1, a package of ab initio programs, http://www.molpro.net (2006).

57. Barbatti, M. et al. The on-the-fly surface-hopping program system Newton-X: Application to ab initio simulation of the nonadiabatic photodynamics of benchmark systems. J. Photochem. Photobiol. A 190, 228-240 (2007).

58. Honig, B., Jortner, J. \& Szöke, A. Theoretical Studies of Two-Photon Absorption Processes. I. Molecular Benzene. J. Chem. Phys. 46, 2714-2727 (1967).

59. Ghafur, O., Siu, W., Johnsson, P., Kling, M. F., Drescher, M. \& Vrakking, M. J. J. A velocity map imaging detector with an integrated gas injection system. Rev. Sci. Instrum. 80, 033110 (2009).

60. Eppink, A. T. J. B. \& Parker, D. H. Velocity map imaging of ions and electrons using electrostatic lenses: Application in photoelectron and photofragment ion imaging of molecular oxygen. Rev. Sci. Instrum. 68, 3477-3484 (1997).

\section{Acknowledgements}

We acknowledge fruitful discussion of these results with Herschel Rabitz and Brett Esry. Augustana College personnel and equipment were funded by the National Science Foundation grant number 0969687 and National Science Foundation/EPSCoR gran number 0903804. JRML operations and personnel (including partial sabbatical leave funding for E.W.) were supported by the Chemical Sciences, Geosciences and Biosciences
Division, Office of Basic Energy Sciences, Office of Science, U.S. Department of Energy. M.F.K., R.d.V.-R. and R.S. are grateful for support by the German Science Foundation via the Cluster of Excellence: Munich Center for Advanced Photonics. B.B. and M.F.K. received partial support from the DFG via the grants Kl-1439/2 and Kl-1439/3.

\section{Author contributions}

E.W., I.B.-I. and M.F.K. designed the experiment. C.E.R., B.B., P.R.A., M.F.K. and E.W. implemented the rapid inversion. C.E.R., P.R.A. and E.W. constructed the computer control for the experiment. The experiment was carried out by M.Z., C.E.R., B.J., P.R.A., U.A., B.G., S.D., K.D.C., I.B.-I. and E.W. The data analysis and interpretation were done by B.J., E.W., M.Z., M.F.K., R.d.V.-R., R.S. and I.B.-I. R.S. and R.d.V.-R. provided theoretical analysis and calculations. E.W., B.J., K.D.C., M.F.K., R.d.V.-R., R.S. and I.B.-I wrote the manuscript, and all authors reviewed the manuscript and offered comments.

\section{Additional information}

Competing financial interests: The authors declare no competing financial interests.

Reprints and permission information is available online at http://npg.nature.com/ reprintsandpermissions/

How to cite this article: Wells, E. et al. Adaptive strong-field control of chemical dynamics guided by three-dimensional momentum imaging. Nat. Comm. 4:2895 doi: $10.1038 /$ ncomms3895 (2013). 